\title{
IoT and AI Based Emotion Detection and Face Recognition System
}

\author{
Pravin Kumar Singh, Mandeep Kaur
}

\begin{abstract}
Human facial emotion detection is a prime goal in the current technical world. Robotic applications are being applied in almost all domains. To enable successful human-robotic interaction, emotion recognition is crucial. This project aims to develop and implement a novel, automatic emotion detection system and facial recognition system based on AI (Artificial Intelligence) and IoT (Internet of Things).
\end{abstract}

Index Terms: Face Recognition, Emotion Detection, Artificial Intelligence, Internet of Things,

\section{INTRODUCTION}

Recent era is an era of technology which includes IoT (Internet of Things) and AI (Artificial Intelligence) being the trending technology in the field of Machine Learning or Robotics.

Man-made intelligence in straightforward words implies Artificial Humans or Intelligent Machines that work or process the information same as people. Computer based intelligence models can be Siri Virtual Assistant on the Apple iPhones, Self-driving autos and so on. People can be recognized with different animals simply because of one of a kind attributes called knowledge. Computerized reasoning is fundamentally used to mechanize things. Any sort of gadgets that is playing out every one of the undertakings independent from anyone else without human intercession, we can state that $\mathrm{AI}$ is executed in that gadget to get working. Artificial intelligence has changed the situation of work done these days. Different organizations are supplanting laborers with $\mathrm{AI}$ empowered robots or robotization innovations.

Rock solid works which were taking various days to be finished, now can be finished in a moment or two or minutes on account of Artificial Intelligence. Presently a day's different undertakings like reconnaissance, information mining, interviews, wars and so forth are finished with the assistance of robots in lieu of people. These AI based robots are all around prepared and clever in previously mentioned territories as it can diminish human setbacks. US based firms are fortifying different kinds of automated armed force to battle in war [1].

Revised Manuscript Received on July 5, 2019.

Pravin Kumar Singh, Computer Science and Engineering, Sharda University, Greater Noida, India,

Dr Mandeep Kaur, Associate Professor at the Department of Computer Science \& Engineering, Sharda University
IoT is a notable innovation, which fundamentally is an associated system of physical articles or gadgets that can be gotten to through internet. IoT regularly utilize sensors to extricate information and exchange the information over system utilizing an IP address. IoT is being utilized one end to the other and is across the board as a wellbeing and security gadget, a health tracker, an expanded and computer-generated reality and so forth.

IoT has been thriving in specialized market and in this manner executed even in modest tasks moreover. Being savvy innovation, each examiner or task researcher favors utilizing IoT gadgets to actualize and execute the undertaking instantly. IoT regularly comprises of different gadgets like sensors and above all Raspberry Pi to work those sensors and concentrate information gathered by those sensors [2].

For instance, different tasks like programmed entryway open frameworks, message ready frameworks, wellbeing ready frameworks, and so on are effectively executed with the assistance of IoT. In entryway open framework, we see that when we draw nearer to the entryway the entryway opens without anyone else's input. This wonder is because of an IoT gadget set that perceives your face and contrasts it and the pictures gave in the database and once a legitimate match is discovered it opens the entryway. On the off chance that the face isn't perceived then it will send a speedy notice to the proprietor of the house in regards to the issue and takes care of the issue rapidly and effectively. This is the means by which really IoT gadgets works together utilizing web association. These innovations are shabby and simple to actualize and can take in a considerable measure of new things by doing different little or huge looks into utilizing IoT [3] [4] [5]

Additionally, these innovations are being utilized in feeling discovery and face acknowledgment or distinguishing proof frameworks utilizing our face. Innovation is growing quicker and quicker which makes us people languid about doing some ordinary errands however now because of these advancements we can skirt those means or assignments and let the gadgets perform them independent from anyone else for us. For instance, composing password which is currently supplanted by face open innovation. [6] 
In Fig 1, we can see different outward appearance of a person taken to break down.

These countenances will be breaking down utilizing the element extraction calculations and distinguish the articulation out of it. At the point when these pictures experience the component extraction process, PC investigates the pictures and changes over them to greyscale configuration to sum up the picture and concentrate the articulations from it.

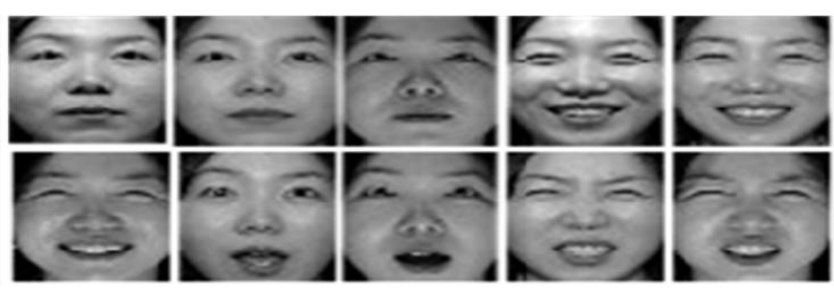

Fig 1: Dataset of Facial Expression

Fig 2 shows the greyscale phase of all the images provided for analysis according to the face lightings [7].

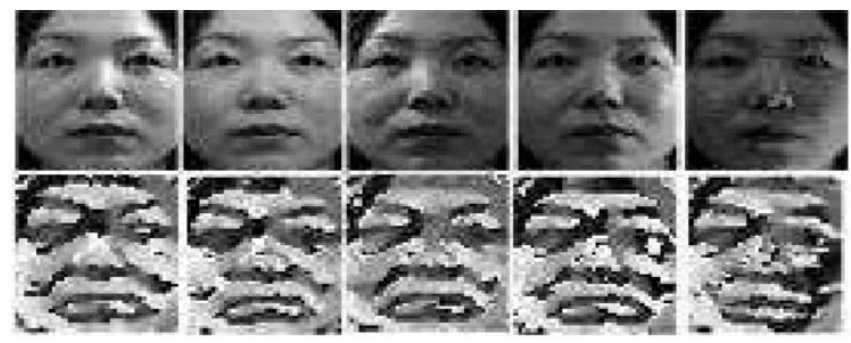

Fig 2: Greyscale Facial Expression

A Basic setup or design of the entire venture is delineated in the fig 3 given underneath. This demonstrates to us how the entire setup will be figured out how to get this venture set up. Typically, a raspberry pi is required and a cloud get to is expected to get the task working with the assistance of a web association with the raspberry pi gadgets. With the assistance the gadgets associated with the raspberry pi web will be exchanged to every one of the gadgets and information can be effectively separated and transferred to the web or the cloud. When the information is transferred it very well may be sent as a caution to different gadgets we require.

This is the means by which IoT based outward appearance location instrument happens. We will utilize a similar plan to get our exploration running in the blink of an eye.

Fig 3, demonstrates the essential engineering of human face acknowledgment and feeling acknowledgment framework. We fundamentally utilize raspberry pi as our base PC and interface every one of the gadgets to it, for example, camera module and so forth. When we turn on the pi, the gadget will get the camera module working utilizing which we will examine the pictures caught by the camera and go it through different calculations to sum up the articulations. These identified articulations will then be sent to a cloud framework and from that point it will be sent to the clients recorded for email gathering identified with the subtle elements gathered. These points of interest can likewise be gotten to utilizing a web stage with legitimate approval leeway. [8]

As should be obvious how rapidly we can gather the information examine the information and exchange the information all around the globe. Furthermore, can likewise rapidly reaction back as we get continuous information examination utilizing the design.

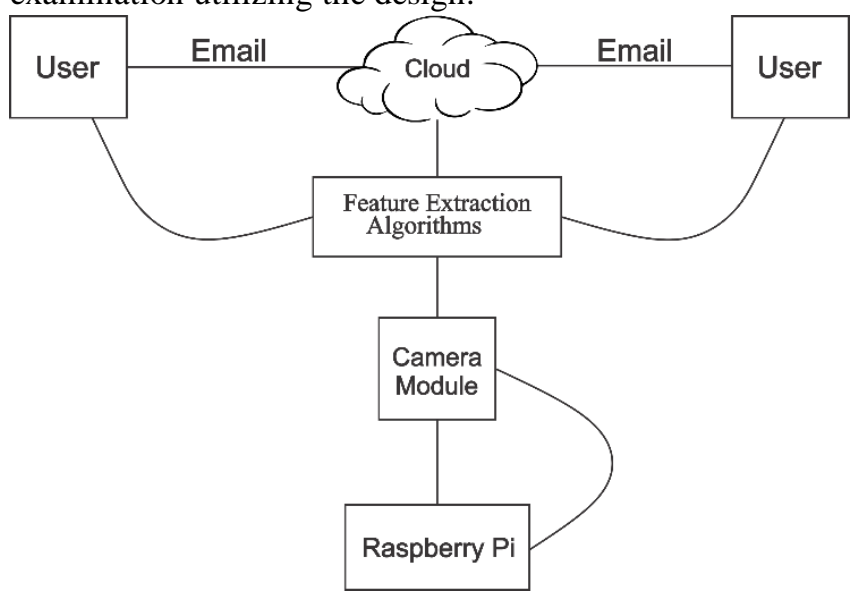

Fig 3: Architecture for Face and Emotion Detection

\section{LITERATURE SURVEY}

\section{Artificial Intelligence without Internet of Things}

The face and emotion identification framework involve different calculations and techniques to achieve its errand. Different scientists have utilized numerous numbers of approaches to accomplish the comparable sorts of results. Some ways are quicker and proficient contrasted with other tedious strategies. The reviews of different face and feeling discovery ventures are being talked about here.

In 2014, FACS (Facial Action Coding System) was used to make a combined human activity based controlling system where all the possible biometric like face recognition gesture recognition and etc. systems can be used to make it a universal system. They had used preprocessing, feature extraction and classification method to obtain the results [9].

In 2015, Datasets of various face and emotion features were used for face and emotion recognition of a person which seemed to be the most efficient method to face and emotion detection. But it comes with various kinds of drawbacks which might include not detecting people of faces that are not provided in the datasets etc. [10].

In 2015, Using MATLAB installed on a PC, face detection and recognition was implemented. Also, PIC16F887 program for door access system was implemented. They have used PCA (Principle Component Analysis) and Viola-Jones Face Detection Method using microcontroller, LCD display, Door Motor, Sensors,

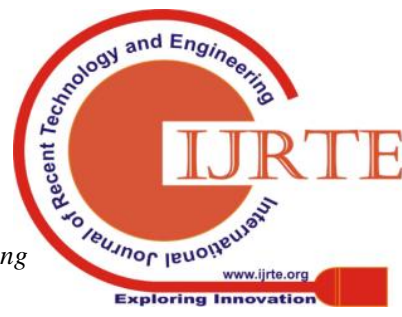


Webcam etc. These all devices will have cost them a lot of money to get this project working which is the main drawback of this project [11].

In 2016, Python programming (OpenCV) library was used to detect faces, feature extraction, emotion recognition, Eye extraction, eyebrow extraction, mouth extraction and emotion classification which is supposed to be the easiest way to achieve human emotions from facial expression. They have talked about facial emotion recognition based on the Image processing and Machine Learning [12].

In 2017, MATLAB was used to detect emotion using face recognition. Methods used by them were feature based approach, holistic approach, hybrid approach and their main objective was to make an application similar to face detection techniques like Viola Jones algorithm and principal component analysis [13].

In 2017, Python 2.8 platform with OpenCV 2.0 Library was used to design an emotion recognition model based on facial recognition in virtual learning environment. The main objective of this research was to introduce a method based on facial recognition to identify students understanding of the entire distance learning process. Methods used by the researchers was feature extraction, subset feature, emotion classifier, a Haar Cascade method is used to detect the input image, a face, as the basis for the extraction of eyes and mouth and then through the sobel edge detection to obtain the characteristic value. The problem in this research was that it doesn't involve the illumination and pose of the image which makes it uncertain how much these factors influence facial expression and thus the final emotion recognition [14].

\section{DRAWBACKS (WITHOUT IOT)}

Past researches were directed for the most part without IoT based modules. Those modules contain different disadvantages and highlight trimmings. Because of no system empowered gadgets joined the information separated from the recognized face couldn't be handled rapidly and effortlessly. It was ordinarily exchanged physically to the territories required.

- High Cost

- Manual Monitoring

- High Power Consumption

- Can't fetch the data online

- More time consumption

- Not portable

\section{SIGNIFICANCES OF IOT}

IOT as we as a whole know is the innovation popular these days and will be the innovation that will change the world in late future likewise as the following mechanical upset. IoT innovation utilizes web empowered gadgets to interface with one another which offers investigating and translation of information progressively.

Various Significances of IoT:

Truly IoT has been helping different enormous organizations to enhance various number of gadgets that are making the lives of individuals less demanding. Thus, IoT has helped us in each conceivable field including Cloud stages, for example, gathering information from the interconnected gadgets and transferring the information to the cloud for which can be gotten to from anyplace on the planet by anybody with right consents. A few focal points of IoT can be seen in: [15]

- Automation and control

- Communication

- Information

- Time

- Better quality of life

\section{Automation and Control}

A. Physically interconnected gadgets can speak with one another remotely without human mediation and can move information in a matter of seconds has changed the method for living. Presently works should be possible considerably more snappy and auspicious conveyance of the outcomes.

\section{Communication}

Communication between two devices also known as Machine to Machine (M2M) communication can transmit data continuously hence clear idea about the communication and better quality.

\section{Information}

As much knowledge we get that much better it gets to make decisions.

\section{Time}

Time is everything is today's world. As IoT helps us get things done as quickly as possible we get more time to get our things done without needing to worry about anything. And in today's fast paced world we could use that extra time.

Better quality of life

All these technologies that are making our daily life so easier sums to a better quality of life.

\section{LITERATURE SURVEY}

\section{Artificial Intelligence with Internet of Things}

As we know clearly about the significances of IoT which motivated us to implement this technology to our project. Making use of IoT in the field of emotion recognition and face detection has changed the way it was used before. Now the collected data can be quickly uploaded to the cloud and analyzed from anywhere in the world.

In 2016, a case study was conducted on the effectiveness of the proposed scheme in detecting "missing" person indicates that the procedure works effectively in the IoT-Cloud Infrastructure. This paper has discussed about the decentralized face recognition scheme for distributed video surveillance in IoT-Cloud Infrastructure [16] [17].

In 2018, using image processing technology to authenticate person to enter in home. For image processing, they have used pi camera module which was attached to Raspberry $\mathrm{Pi}$ which aids to store various faces in the databases. When someone wants to enter in home, he should stand in front of the camera. Camera will then recognize the face and compare with the faces stored in the LFW database. This system normally performs the 
detection and recognition rapidly in real time. This project utilizes the basic web cam and the internet connection to create a door that unlocks itself via facial recognition [18].

By implementing IoT in the projects various researchers have found that it is much easier and safer way to use the received data from the face and emotion recognition system. The data can be reanalyzed anytime from anywhere using the cloud infrastructure. Similarly, in the field of health and personal health we can make use of AI and IoT in really effective manner. With IoT devices we can collect the health information of a person and securely upload the data to the cloud and allow access to the data to specific persons such as personal doctors, parents or guardians of any kind.

\section{Comparison of Projects based on IoT and without IoT}

There is a very broad difference between projects conducted under IoT and without IoT. After doing a complete literature survey in face and emotion detection using IoT and without IoT devices, we have found various reasons to implement IoT in our project.

- Projects without IoT are very costly whereas projects with IoT are comparatively cheaper.

- Projects without IoT are time consuming whereas projects with IoT provide results in real time which make a big amount of difference.

- Projects without IoT have a performance percentage of $72.65 \%$ whereas performance percentage of projects with IoT is $98 \%$ [19].

In Fig 4, we have a bar chart to represent projects with and without IoT and their performance conducted in various years. The chart clearly shows the analysis report and their percentage success rate [20].

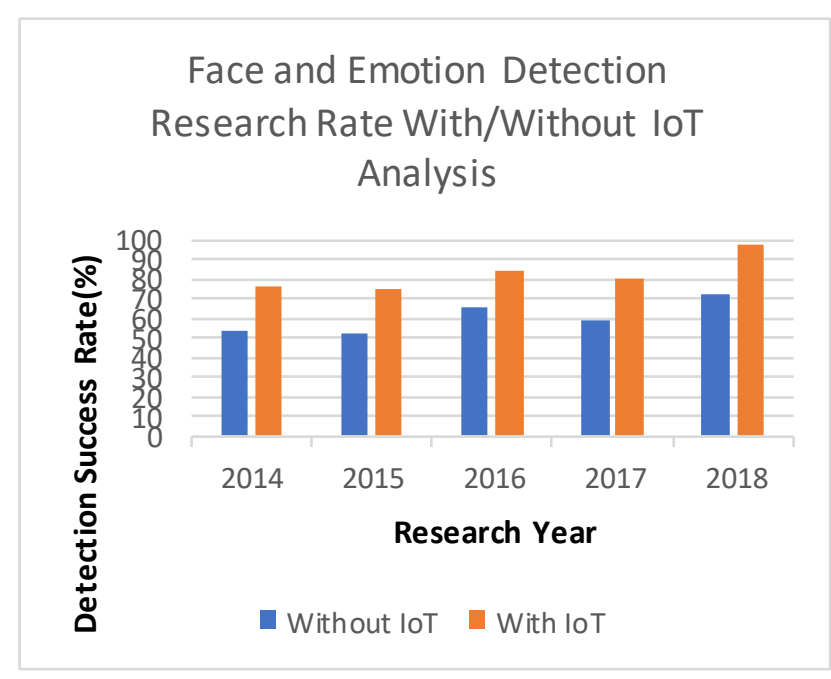

Fig 4: Face and Emotion Detection Bar Chart

\section{CONCLUSION}

The principle goal of this investigation is to examine the significances of IoT in the field of emotions and face recognition. The fundamental thought of utilizing IoT and AI for face and feeling acknowledgment. We demonstrated a review of existing Face and Emotion acknowledgment frameworks and talked about the utilization of Feature extraction in face and feeling acknowledgment framework. The face and its feelings are recognized and removed utilizing different techniques and calculations like Viola Johns calculation, Feature extraction, PCA (Principle Component Analysis), SVM (Support Vector Machine), emotion classifier, a Haar Cascade strategy, extraction of eyes and mouth, eyebrows and so forth. In light of the examination results, highlight extraction and arrangement has turned out to be the best procedure contrasted with different systems utilized in their exploration ventures.

\section{REFERENCES}

1. Greg Allen, Taniel Chan, "Artificial Intelligence and National Security", July 2017

2. James Manyika, Michael Chui, Peter Bisson, Jonathan Woetzel, Richard Dobbs, Jacques Bughin, Dan Aharon, “The Internet of Things: Mapping the Value Beyond the Hype", June 2015

3. I.Yugashini, S.Vidhyasri, K.Gayathri Devi, "Design and Implementation of Automated Door Accessing System With Face Recognition", International Journal of Science and Modern Engineering (IJISME), Volume 12, November 2013

4. Shrikrushna Khedkar, Dr. G.M. Malwatkr. 2016. Survey on Home Automation using Raspberry Pi through GSM. IJSR. 5(1), ISSN: 2319-7064.

5. SHaik Anwar, D. Kishore,"IoT based Home security system with alert and door access control using Smart Phone", December 2016, IJERT.

6. Anagha S. Dhalvikar, Dr R.K.Kulkarni "Face detection and facial expression recognition System", 2014 International conference Mumbai.

7. Leandro y. manoa, bruno s. faical, luis h. v. nakamuraa, pedro h. gomese, giampaolo 1. libralonc, rodolfo i. menegueteb, geraldo p. r. filhoa, gabriel t. giancristofaroa, gustavo pessin, bhaskar krishnamachari, j'o ueyama, "Exploiting IoT Technologies for Enhancing Health Smart Homes through patient identification and emotion recognition”, March 2016

8. Prof Archana Gaikwad, Prof. Paresh D. Sonawane "An efficient video surveillance system using video-based face recognition on Real world Data", IJSETR, Volume 5, Issue 4.

9. Anuradha Savadi, Chandrakala V Patil, "Face Based Automatic Human Emotion Recognition", IJCSNS International Journal of Computer Science and Network Security, VOL.14 No.7, July 2014

10. Bharati A.Dixit and Dr. A.N.Gaikwad "Statistical Moments Based Facial Expression Analysis" IEEE International Advance Computing Conference (IACC), 2015

11. Hteik Htar Lwin, Aung Soe Khaing, Hla Myo Tun, "Automatic Door Access System Using Face Recognition”, International Research Journal of Engineering and Technology (IRJET), Volume 4, Issue 06, June 2015

12. Monika Dubey, Prof. Lokesh Singh, "Automatic Emotion Recognition Using Facial Expression”, International Research Journal of Engineering and Technology (IRJET), Volume 3, Issue 02, February 2016

13. Prof. Neelum Dave, Narendra Patil, Rohit Pawar, Digambar Pople, "Emotion Detection Using Face Recognition", IJESC, Volume 7, Issue No.4, 2017

14. D. Yanga, Abeer Alsadoona, P.W.C. Prasad, A. K. Singhb, A. Elchouemic, "An Emotion Recognition Model Based on Facial Recognition in Virtual Learning Environment", 6th International Conference on Smart Computing and Communications, ICSCC 2017 7-8 December 2017

15. Biswanath saha, "IoT Platform and its significances", https://www.mobiloitte.com/blog/iot-platform-and-its-significance/, july 4, 2017

16. Ravi Kishore Kodali, Vishal Jain, Suvadeep Bose and Lakshmi Boppana" IoT Based Smart Security and Home Automation System", IEEE 2016

17. S.V. Thate, A.S. Narote, S.P. Narote, "Human face Detection and Recognition in Videos", 21-24 September 2016, Jaipur, India.

18. Sandesh Kulkarni, Minakshee Bagul, Akanksha Dukare, Prof. Archana Gaikwad, "Face Recognition using IoT", International Journal of Innovations \& Advancement in Computer Science (IJIACS), ISSN 2347 - 8616, Volume 7, Issue 3, March 2018

19. Martinez, A., Du, S., "A model of the perception of facial expressions of emotion by humans: Research overview and perspectives", Journal of Machine Learning Research 13, 1589-1608, 2012. 
20. John A. Stankovic" Research Direction for the Internet of Things", February 2014, University of Virginia

21. S.V. Thate, A.S. Narote, S.P. Narote, "Human face Detection and Recognition in Videos", 21-24 September 2016, Jaipur, India.

\section{AUTHORS PROFILE}

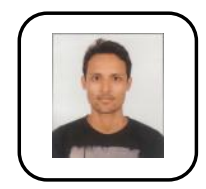

Pravin Kumar Singh, M.Tech in Computer Science and Engineering (Software Engineering), Certification of Certified Ethical Hacker from EC-Council.

Dr. MandeepKaur is working as an Associate Professor at the Department of Computer Science \& Engineering, Sharda University. She has completed her B.Tech in CSE from UPTU, M.Tech in CSE from PTU \& completed Ph.D from Sharda University. Her topic of research was "Thought Recognition: Knowledge Discovery from EEG Signals and Classifying by Classifier Ensemble". She is M.Tech (Project \& Dissertation) Coordinator cum Mentor and guiding research investigations for under-graduate, post-graduate engineering students \& Ph.D scholars in the areas including Image \& Signal Processing, Machine Learning, Neural Networks etc. She has 13 years of Teaching. She has published 03 book chapters in Springer \& WSEAS Press and also authored more than 40 research papers in various reputed International / National Journal and Conferences and attended more than 20 FDPs, Workshops, Seminars and Conferences. 